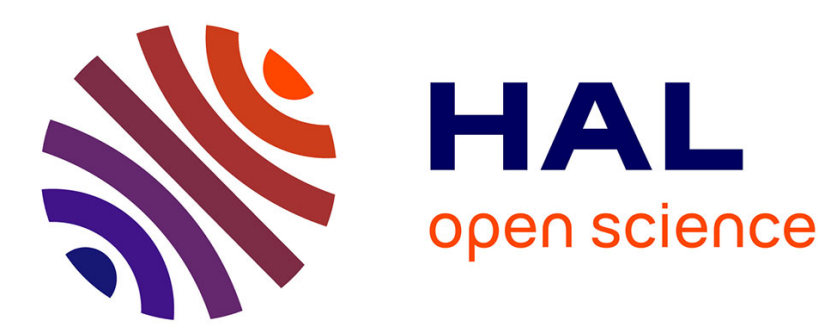

\title{
Précarité et troubles psychologiques: l'effet médiateur de la perspective temporelle
}

\author{
Nicolas Fieulaine, Thémis Apostolidis, Fabien Olivetto
}

\section{To cite this version:}

Nicolas Fieulaine, Thémis Apostolidis, Fabien Olivetto. Précarité et troubles psychologiques: l'effet médiateur de la perspective temporelle. Les cahiers Internationaux de Psychologie Sociale, 2006, 72, pp.51-64. halshs-00408113

\section{HAL Id: halshs-00408113 https://shs.hal.science/halshs-00408113}

Submitted on 1 Oct 2009

HAL is a multi-disciplinary open access archive for the deposit and dissemination of scientific research documents, whether they are published or not. The documents may come from teaching and research institutions in France or abroad, or from public or private research centers.
L'archive ouverte pluridisciplinaire HAL, est destinée au dépôt et à la diffusion de documents scientifiques de niveau recherche, publiés ou non, émanant des établissements d'enseignement et de recherche français ou étrangers, des laboratoires publics ou privés. 


\title{
Précarité et troubles psychologiques: l'effet médiateur de la perspective temporelle
}

\author{
Nicolas FIEULAINE ${ }^{12}$, Thémis APOSTOLIDIS ${ }^{23}$ et Fabien OLIVETTO \\ 1. Groupe d'Étude des Relations Asymétriques (GÉRA), Université Lumière Lyon 2, Bron, France \\ 2. Laboratoire de Psychologie sociale, Université de Provence, Aix-en-Provence, France \\ 3. Épidémiologie \& Sciences Sociales Appliquées à I'Innovation Médicale, I.N.S.E.R.M. (U-379)
}

\begin{abstract}
Résumé : L'objectif de cette étude est d'analyser le rôle joué par le rapport au temps dans le vécu des situations de précarité, au travers de l'étude des régulations sociales de la Perspective Temporelle (PT) et du rôle de cette variable psychosociale dans les liens entre situations de précarité et troubles psychologiques. L'analyse des données recueillies auprès de 275 consultants d'un centre d'examen de santé fait apparaître les régulations sociales dont la PT fait l'objet, ainsi que les liens que le rapport au passé entretient à la fois à la précarité et aux troubles psychologiques (troubles anxieux, humeur dépressive). Enfin, les résultats suggèrent le rôle médiateur joué par le rapport négatif au passé dans l'émergence des troubles psychologiques liés aux situations de précarité.
\end{abstract}

Les liens entre santé et précarité représentent à l'heure actuelle un des enjeux majeurs dans le champ des politiques de santé publique en France et en Europe (European Foundation for the Improvement of Living and Working Conditions, 1997). De nombreux travaux ont souligné l'impact de la précarité sur l'état de santé physique ainsi que son rôle dans l'augmentation de la prévalence des troubles psychologiques (cf. Lebas et Chauvin, 1998). Face à la diversité des situations que recouvre la notion de précarité, ces questions ont suscité de nombreux travaux multidisciplinaires. Ceux-ci se sont attachés à la fois à parvenir à une définition opérationnelle de ce phénomène, à mettre en évidence les variables sociales, psychosociales et psychologiques qui y sont liées, ainsi qu'à étudier son impact sur le bien-être et la souffrance psychologique.

\section{Les situations de précarité}

Des nombreux travaux sociologiques visant à spécifier et à caractériser les situations de précarité se dégagent un certain nombre de constats. La précarité apparaît comme un ensemble de situations multifactorielles et dynamiques, qui s'établissent en lien avec les trajectoires des individus et des groupes (Bouchayer et Verger, 1994). L'émergence des nouvelles formes d'emploi dites «atypiques » (définies par leur écart à la norme que représente le contrat à durée indéterminée et à temps plein; i.e. contrat à durée déterminé, intérim, temps partiel, ...), ainsi que l'apparition corrélative de "travailleurs pauvres » ont en effet institutionnalisé une double déstabilisation, économique et sociale (Appay, 1997). Dépassant largement les frontières du monde du travail où ils ont trouvé leur source, les processus de précarisation ont un impact sur de nombreuses dimensions des vécus individuels et sociaux. Cet effet de déstabilisation qu'entraînent les situations marquées par la fragilisation des statuts sociaux est considéré comme se situant au cœur de ce que l'on nomme les situations de précarité. Il en découle que la précarité se définit aujourd'hui comme un ensemble de situations de privation et de fragilisation où s'articulent l'instabilité des trajectoires, la difficulté d'anticiper l'avenir et la désaffiliation sociale.

\section{L'impact de la précarité sur la santé}

Marquées par l'instabilité et l'incertitude, les situations de précarité ont un impact sur l'état de santé. De nombreux travaux ont souligné l'émergence d'inégalités de santé liées aux phénomènes de précarisation (Lebas et Chauvin, 1998), et les liens entre situations de précarité et mauvais état de santé ont été à de multiples reprises démontrés (Foubert, Chauvin et Facy, 2001). La santé des populations précaires apparaît non pas comme présentant des caractéristiques spécifiques, mais comme représentant une concentration de problèmes observés en population générale

Pour toute correspondance relative à cet article, s'adresser à Nicolas Fieulaine, Université Lumière Lyon 2, Institut de Psychologie, GÉRA, 5 avenue Pierre Mendès-France, 69676 Bron CEDEx, France ou par courriel à <nicolas.fieulaine@univ-lyon2.fr>.

Nicolas Fieulaine a assuré l'analyse des données et rédigé l'article, Thémis Apostolidis a supervisé la conception de l'étude, co-rédigé l'article et participé aux différentes phases du travail et Fabien Olivetto a élaboré le questionnaire et effectué le recueil des données. 
(La Rosa, 1998). Une large étude, comparant l'état de santé de publics précaires et non-précaires (ibid.) fait apparaître une plus forte morbidité diagnostiquée et-ou déclarée chez les populations en situation de précarité. Celles-ci apparaissent également comme ayant moins de pratiques préventives (activité physique, dépistages, vaccinations, recours aux soins, ...), plus de pratiques à risque (consommation de tabac et d'alcool), un moindre recours et un plus mauvais accès aux soins. Enfin, concernant les troubles psychologiques (dépression, anxiété), plusieurs enquêtes ont mis en évidence leur plus forte prévalence dans les populations en situation de précarité (Kovess-Masféty, 2001 ; La Rosa, Consoli, Le Clésiau, Birouste, Foubert et Soufi, 2000).

\section{Précarité et santé : le rôle des facteurs psychologiques}

Si les liens entre précarité et état de santé semblent solidement fondés, ils s'établissent néanmoins de manière complexe. Les situations de précarité sont ainsi difficilement spécifiables uniquement au travers des indicateurs socio-économiques objectifs. Il apparaît nécessaire de considérer le rapport subjectif que les individus entretiennent à ces situations, le sens qu'ils attachent à leur appartenance sociale, afin de comprendre la pluralité des vécus et leurs conséquences sur la santé. Ainsi, les analyses de Paugam (2000) concernant la précarité salariale mettent en évidence que, plus qu'aux catégories socioprofessionnelles, l'état de santé physique et psychologique se trouve lié au rapport subjectif que les individus entretiennent à l'emploi précaire. Par ailleurs, certains travaux ont suggéré l'intervention de différentes variables de personnalité (efficacité personnelle, estime de soi) dans le lien entre précarité et santé physique ou psychique, entraînant une hétérogénéité des modes de faire face, et ainsi des impacts différenciés sur l'état de santé (Cazals-Ferré et Llorca, 2002 ; Sordes-Ader et Tap, 2002). Les résultats établis amènent ainsi à souligner que la relation entre situations de précarité et état de santé n'est ni linéaire, ni immédiate. Il apparaît dès lors intéressant d'analyser le rôle joué par les variables psychologiques qui peuvent constituer des vecteurs de protection ou de vulnérabilisation face aux situations de précarité. Ainsi, ces variables pourraient agir comme des médiateurs entre les insertions sociales précaires et leurs conséquences sur la santé, notamment psychologique. Cette piste de travail posant le principe d'une médiation pourrait ainsi renseigner sur le mécanisme génératif par lequel s'établit la relation entre situations de précarité et troubles psychologiques. C'est-à-dire qu'elle peut permettre d'explorer le «pourquoi » ou le « com- ment » de cette relation au travers du rôle joué par différentes variables médiatrices (cf. Frazier, Tix et Barron, 2004).

\section{Précarité et rapport au temps}

Parmi les facteurs importants dans l'analyse du vécu des situations de précarité, il a été mis en avant l'intérêt de considérer le rapport au temps (SinghManoux et Marmot, 2005). En effet, au-delà des critères objectifs d'insertion sociale (emploi, revenus), l'incertitude et l'instabilité qui marquent les situations précaires peuvent entraîner des rapports spécifiques au temps. Ainsi, le rapport à l'avenir et la difficulté à l'anticiper ont été identifiés comme des dimensions importantes pour comprendre les logiques d'action en situation de précarité (Billiard, Debordeaux et Lurol, 2000). Même si le rapport à l'avenir constitue un facteur essentiel, le rapport au temps ne peut être analysé seulement au travers de cette dimension. Le rapport au passé apparaît lui aussi fondamental, la « fragilité biographique » qui peut accompagner les trajectoires sociales en situation de précarité constituant un facteur de vulnérabilisation (Delors et Hubert, 2000). Ce postulat de l'importance du temps psychologique repose également sur le fait qu'il correspond à une dimension fondamentale du rapport que les individus entretiennent à leur situation sociale (Allport, 1948). Les analyses concernant l'impact du chômage sur les troubles psychologiques ont à cet égard souligné et mis en évidence le rôle central joué par le rapport au temps (e.g. Fahoda, 1982 ; Feather, 1997). Ainsi, parmi les variables susceptibles d'intervenir dans le lien entre situations de précarité et troubles psychologiques, le rapport au temps apparaît particulièrement pertinent. Ces constats empiriques conduisent à considérer la possibilité que ce soit notamment au travers de «troubles temporels » (Sordes-Ader et Tap, 2002) que se génèrent ou s'alimentent le mal-être et la souffrance psychologiques.

\section{La Perspective Temporelle : une variable médiatrice?}

Lewin (1942) a posé les bases d'une approche sociocognitive du temps psychologique au travers de la notion de « perspective temporelle » $(\mathrm{PT})$. Selon lui, l'espace de vie d'un individu ne se limite pas à ce qu'il considère dans la situation présente, mais inclut aussi le futur, le présent et le passé. Lewin considère que les actions, les émotions et le moral d'un individu à chaque instant sont sous la dépendance de cette perspective temporelle totale. Il définit la PT comme « la totalité des points de vue d'un individu à un moment donné sur son futur psychologique et 
sur son passé psychologique » (1951, p. 75). La notion de perspective englobe principalement les idées de points de vue, de direction, de contexte renvoyant aux trois registres temporels qui déterminent la conduite d'un individu. C'est-à-dire comment, de manière interdépendante, le flux de l'expérience est-il cognitivement rattaché à des catégories temporelles et quel impact ce marquage temporel exerce sur l'expérience (Zimbardo et Boyd, 1999). L'approche de Lewin a permis de dépasser l'opposition entre temps subjectif et objectif, au bénéfice d'un temps relatif, fonction des rapports dynamiques qui s'établissent entre les individus et leur environnement. Le construit psychologique que représente la PT désigne ainsi le rapport que les individus et les groupes entretiennent au présent, au passé et au futur, à la fois au niveau de l'importance accordée à chaque registre temporel, ainsi que de l'attitude qui leur est rattachée. Cette multidimensionnalité de la PT est fondamentale, la nécessité de considérer simultanément les différents registres temporels (passé, présent et futur) ayant été plusieurs fois soulignée et mise en évidence (e.g. Fingerman et Perlmutter, 2001). La prise en compte de la PT dans l'étude des liens entre précarité et santé psychologique est d'autant plus pertinente que celle-ci est apparue liée d'une part aux insertions sociales et d'autre part aux comportements et perceptions dans le champ de la santé, notamment psychologique.

Bien que la question de la régulation sociale de la PT soit restée en retrait des travaux au profit d'une approche centrée sur les différences interindividuelles, il a été montré que la PT varie en fonction des variables sociodémographiques (sexe, âge, niveau de diplôme, emploi, classe sociale: Lamm, Schmidt et Trommsdorf, 1976; D'Alessio, Guarino, DePascalis et Zimbardo, 2003). Dans une approche psychosociale, l'analyse des variations sociales et culturelles de la PT apparaît d'autant plus fondamentale que celle-ci est tributaire de la socialisation (Stein, Sarbin et Kulik, 1968), et qu'elle s'établit dans un rapport circulaire aux expériences des individus et des groupes. En effet, la PT est susceptible d'être affectée par certaines expériences (guerres, maladies, traumatismes, chômage...), mais ces expériences prennent en même temps leur sens au travers de la PT dans laquelle se situent les individus (Lewin, 1942). Ainsi, la P'T participe aux stratégies d'ajustement face aux conditions d'existence et aux évènements (Epel, Bandura et Zimbardo, 1999). Elle peut donc représenter une variable agissante dans le rapport que les individus entretiennent à leurs insertions sociales, et par-là devenir un médiateur potentiel de l'impact de ces situations, notamment sur l'état de santé psy- chologique (troubles anxieux, humeur dépressive). Cette hypothèse apparaît d'autant plus justifiée que le lien entre PT et santé psychologique a été de nombreuses fois établi (cf. Bonizeell et Zimbardo, 2004). En particulier, la PT est apparue comme un facteur important associé aux troubles psychiques comme l'anxiété ou la dépression (Krauss, 1967 ; Zimbardo et Boyd, 1999) et plus globalement à la souffrance psychologique. Parmi ces contributions, il a été montré que la PT est un construit agissant dans l'émergence ou la gravité de la détresse psychologique liée aux expériences traumatiques (rumination négative du passé, Holman et Silver, 1998). Ce qui suggèrerait dans le cadre de notre problématique l'intérêt de considérer le rôle joué par la perspective temporelle pour comprendre comment la précarisation peut altérer le rapport à soi sur le plan psychologique. Cette hypothèse de la PT comme variable médiatrice est appuyée par les observations de plusieurs recherches longitudinales montrant que l'impact de la perte d'emploi sur la souffrance psychologique s'établit au travers du rapport au temps (Wanberg et Griffiths, 1997; Waters et Muller, 2003).

\section{Objectifs}

Dans ce cadre, l'objectif général de cette étude est d'étudier le rôle joué par la PT dans les liens entre insertions sociales précaires et troubles psychologiques. Cet objectif implique une démarche en plusieurs étapes.

- Dans un premier temps, il s'agit d'étudier les variations de la PT en fonction des insertions sociales, et plus particulièrement vérifier les liens suggérés dans la littérature entre situations de précarité et PT.

- Ensuite, il s'agit mettre en évidence les liens précédemment établis ou envisagés, d'une part, entre précarité et troubles psychologiques, et, d'autre part, entre PT et troubles psychologiques.

- Enfin, dans un troisième temps, il s'agit de tester le rôle médiateur de la PT dans les liens observés entre précarité et troubles psychologiques. Cette étape permettra d'étudier si la PT peut être considérée comme un vecteur de vulnérabilisation etou de protection par rapport aux troubles psychologiques dans les situations de précarité.

\section{Méthode}

\section{Population et procédure}

L'étude s'est déroulée au Centre d'Examens de Santé (CES) de la Caisse Nationale d'Assurance Maladie (CNAMTS) à Marseille entre juin et sep- 
tembre 2003. Les CES sont chargés d'assurer les bilans de santé gratuits périodiques auxquels a droit tout assuré social du régime général, ainsi que ses ayants droits (soit environ $90 \%$ de la population française). D'autre part, ces Centres sont chargés de promouvoir et d'assurer les examens de santé auprès des populations en situation de précarité. Dans le CES de Marseille, les statistiques de fréquentation montrent que les personnes en situation de précarité représentent environ $43 \%$ des personnes reçues.

Le recueil a été effectué à l'occasion d'une recherche réalisée à la demande du CES, visant à connaître les profils des consultants âgés de 25 à 44 ans ainsi que leurs opinions à l'égard des modalités d'accueil et de prise en charge. La procédure de recrutement a été conçue en fonction des modalités d'accueil du Centre. Tous les consultants remplissent à l'accueil le questionnaire administratif du CES, sont ensuite soumis à des prélèvements urinaires et sanguins et patientent alors dans une salle d'attente avant l'entretien avec le médecin. C'est à l'occasion de cette attente que les consultants ont été sollicités pour participer à l'enquête à partir du critère d'inclusion concernant la tranche d'âge visée. Ce renseignement était fourni par l'étiquette «consultant» du dossier transmis par l'accueil. Annoncée comme portant sur la santé des 25-44 ans, l'enquête a été présentée comme étant indépendante du processus d'examen en cours et la participation comme volontaire, individuelle et strictement anonyme. Après avoir rempli le questionnaire, les participants étaient invités à le déposer à l'accueil une fois la visite médicale terminée. À cette occasion, un numéro de dossier était associé provisoirement à chaque questionnaire de façon à y rapporter un score de précarité correspondant (score ÉPICES calculé à partir de la saisie du questionnaire CES de chaque consultant, $c f$. infra). Afin de garantir les principes de confidentialité et d'anonymat, une fois cette information prélevée, toute référence au numéro de dossier était immédiatement supprimée.

Au total 447 consultants ont été contactés durant la période de recueil, et 344 ont accepté de participer à l'enquête (soit un taux de refus de $23 \%$ ). Parmi les questionnaires recueillis, 69 étaient inexploitables car partiellement remplis. Concernant ces questionnaires, on peut observer qu'ils émanent pour $72,5 \%$ d'entre eux de personnes identifiées comme "précaires » au regard du score ÉPICES. Par ailleurs, nous n'observons pas de différence en fonction du sexe. L'échantillon final est constitué de 275 personnes $\left(m_{\text {age }}=34.04 ; \sigma=6.42\right), 133$ hommes et 142 femmes.

\section{Instruments de recueil et construction des indicateurs}

\section{Insertions sociales et indicateur de précarité}

Afin de spécifier les insertions sociales des participants, nous avons recueilli leur niveau de diplôme, leur statut par rapport à l'emploi, leur profession et leur situation par rapport au logement. La dimension relationnelle a également été prise en compte au travers de deux questions concernant le fait d'être en couple et d'entretenir des relations régulières avec sa famille. D'autre part, le niveau de précarité a été évalué à partir d'un indicateur de précarité élaboré et validé sur un échantillon national de 197389 consultants des CES : le score ÉPICES (Évaluation de la Précarité et des Inégalités de santé pour les CES; Bihan, Laurent, Sass, Nguyen, Huot, Moulin, Guegen, LeToulemin, LeClésiau, LaRosa, Reach, et Cohen, 2005; Sass, Dupré, Dauphinot, Labbe, Guéguen, Gerbaud et Moulin, 2005). Cet indicateur repose sur une mesure multidimensionnelle de la précarité comprenant 11 questions dichotomiques (le recours à l'aide sociale, l'assurance maladie complémentaire, le logement, le statut conjugal, les pratiques sportives, culturelles et de loisir, les difficultés financières rencontrées, la possibilité de soutien matériel et d'hébergement de la part de l'entourage, et les relations familiales). À partir d'un travail préalable de construction, les 11 items de cet indicateur ont été sélectionnés sur la base de l'analyse des réponses à 42 questions portant sur 5 principaux domaines en jeu dans la définition multifactorielle de la précarité : la situation socio-économique, le logement, la situation vis-à-vis de l'emploi, les diplômes, l'état de santé (Guéguen, Sass et ÉPICES Working Group, 2005). Les réponses sont pondérées différemment en fonction de l'importance de la dimension visée, et le score obtenu constitue l'indicateur de référence pour définir les consultants en situation de précarité dans les CES. Il permet d'une part d'avoir une mesure continue du niveau de précarité (score ÉPICES allant de 0 « niveau de précarité minimum » à 100 « niveau de précarité maximum »), et d'autre part, une valeur seuil (score ÉPICES $=40.2$ ) permettant la classification des consultants en deux groupes, "précaires » et «non précaires ». Sur la base de ce dernier critère, parmi les 275 consultants de notre échantillon, 140 appartiennent au groupe "précaires » et 135 au groupe «non précaires ». La pertinence de cet indicateur apparaît confirmée dans cet échantillon par les variations dont le score ÉPICES fait l'objet en fonction des insertions sociales des personnes interrogées. À titre d'exemple, ce score apparaît plus faible chez les actifs ( $m=29.95, \sigma=21.77$ ) que chez les chômeurs $(m=52.69, \sigma=21.44, F(1,216)=60.30, p<.0001)$, et décroît en fonction du niveau d'étude (aucun di- 
plôme : $m=69.63, \sigma=14.58$; inférieur au bac $: m$ $=47.30, \sigma=24.13$; bac $: m=42.94, \sigma=22.48$; supérieur au bac: $m=31.38, \sigma=20.83 ; F(3,248)$ $=25.93, p<.0001$ ).

\section{Perspective Temporelle}

La PT a été mesurée à partir d'une version courte de la ZTPI (Zimbardo Time Perspective Inventory, cf. Zimbardo et Boyd, 1999; Apostolidis et Fieulaine, 2004 pour la validation de la version française). La ZTPI est une échelle multidimensionnelle auto-administrée permettant de mesurer la PT par rapport aux trois registres temporels et à l'attitude à l'égard de chacun d'entre eux. Elle est construite de manière à prendre en considération les aspects motivationnels, émotionnels, cognitifs et sociaux au travers d'un inventaire de propositions temporellement marquées. Cette échelle dans sa version française est composée de 54 items, indicateurs de cinq dimensions croisant les trois registres temporels et l'attitude à leur égard : La dimension « Passé positif » (attitude positive à l'égard du passé ; e.g. «Le fait de penser à mon passé me donne du plaisir ») ; La dimension "Passé négatif » (vision négative du passé ; e.g. «Je pense aux mauvaises choses qui me sont arrivées dans le passé »); La dimension « Présent fataliste » (attitude fataliste et résignée face à la vie; e.g. «Puisque ce qui doit arriver arrivera, peut importe vraiment ce que fais »); La dimension « Présent hédoniste » (attitude hédoniste et de prise de risque ; e.g. « C'est important de mettre de l'excitation dans ma vie ») et la dimension « Futur » (position tournée vers l'avenir et vers l'accomplissement des buts; e.g. "Je fais aboutir mes projets à temps, en progressant étape par étape »). Les sujets sont invités à répondre à chaque item à l'aide d'une échelle de type Likert sémantisée en cinq points : (1) Cette proposition n'est pas du tout caractéristique de moi- Ne s'applique pas du tout à moi ; (5) Cette proposition est tout à fait caractéristique de moi - S'applique tout à fait à moi.

La spécificité de la population visée (en partie composée de personnes en situation de grande précarité), nous a amené à utiliser une version réduite de la ZTPI, mesurant à partir de 25 items seulement les mêmes 5 dimensions que l'échelle originale (Passé positif, Passé négatif, Présent fataliste, Présent hédoniste, Futur). Cette version a été élaborée après l'examen approfondi des propriétés psychométriques de l'échelle complète validée en français (consistance interne, saillance des items dans la dimension), et en sélectionnant les items les plus représentatifs. Les 25 items retenus ( $f$. Annexe) ont fait l'objet d'une sélection multicritères, en fonction de leurs qualités psychométriques (saturations, communautés) et de leur représentativité qualitative. La validité de contenu de cette version courte a été vérifiée dans cet échantillon au travers d'une analyse factorielle confirmatoire. Les résultats font apparaître un ajustement acceptable des données au modèle prévu en cinq facteurs $\left(\chi^{2}(265)=648.3, \chi^{2} / D L\right.$ $=2.4$, RMSEA $=.07)$. Les consistances internes des sous-échelles de la version courte apparaissent acceptables, même si elles sont plus faibles que pour la version longue (Passé positif : $n=7, \sigma=.64$; Passé négatif : $n=5, \sigma=.73$; Présent fataliste $: n=4, \sigma=$ .65 ; Présent hédoniste $: n=3, \sigma=.73$; Futur $: n=$ $6, \sigma=.65)$.

\section{Troubles psychologiques}

Les troubles psychologiques ont été évalués à l'aide de l'échelle HAD (Hospital anxiety and depression scale; Lépine, 1993 pour la version française). Il s'agit d'un questionnaire auto-administré, qui permet d'évaluer les dimensions d'anxiété et de dépression dans des populations non-psychiatriques. Il est constitué de deux sous échelles de 7 items chacune, qui donnent deux scores mesurant d'une part l'anxiété (HAD-A : peur, sentiments d'appréhension et d'inquiétude ; e.g. «je me fais souvent du souci ») et d'autre part la dépression (HAD-D : tristesse et chagrin, état mélancolique ; e.g. «j'ai perdu l'intérêt pour mon apparence ») à partir de la fréquence rapportée de différents états émotionnels (réponses sur des échelles de type Likert en 4 points). Comme le montrent différentes méta-analyses, les propriétés psychométriques de l'instrument ainsi que la validité des construits mesurés ont été largement confirmées par plusieurs enquêtes, notamment en population générale. Les consistances internes de deux sous-échelles (HAD$\mathrm{A}: n=7, \alpha=.75$; HAD-D $: n=7, \alpha=.70)$ ainsi que la corrélation entre elles $(r=.65, p \leq .01)$ apparaissent satisfaisantes dans cet échantillon au regard des valeurs de référence $(\alpha>.60, .45<r<.75)$ (e.g. Bjelland, Dahl, Haug et Neckelmann, 2002).

\section{Résultats}

\section{Caractéristiques démographiques}

\section{et différences en fonction du sexe}

Les caractéristiques sociodémographiques de l'échantillon ainsi que les valeurs pour les principales mesures sont présentées dans le tableau 1. On constate d'une part que la proportion de chômeurs et de " précaires » est importante, conformément à ce qui était attendu au regard du lieu où ont été effectuées les passations. D'autre part, on constate 
Tableau 1 : Caractéristiques sociodémographiques et valeurs pour les principales mesures

\begin{tabular}{|c|c|c|}
\hline Variables & Hommes & Femmes \\
\hline $\mathcal{N}$ & 133 & 142 \\
\hline$\hat{A g e}(m, \sigma)$ & $34.5(6.11)$ & $33.5(6.68)$ \\
\hline \multicolumn{3}{|l|}{ Niveau de diplôme (\%) } \\
\hline Aucun & 12 & 9.2 \\
\hline Inférieur au bac & 26.3 & 24.6 \\
\hline $\mathrm{Bac}$ & 12.8 & $21.1 *$ \\
\hline Supérieur au bac & 39.1 & 38 \\
\hline Manquants & 9.8 & 7.1 \\
\hline \multicolumn{3}{|l|}{ Statut professionel (\%) } \\
\hline Chômeur & 42 & 39 \\
\hline Salarié & 40 & 38 \\
\hline Inactif & 18 & 23 \\
\hline \multicolumn{3}{|l|}{ CSP (\% chez salariés) } \\
\hline Professions supérieures & 49.1 & $14.8^{* * *}$ \\
\hline $\begin{array}{l}\text { Professions } \\
\text { intermédiaires }\end{array}$ & 22.6 & $44.5^{* * * *}$ \\
\hline Professions inférieures & 24.5 & $37^{*}$ \\
\hline Manquants & 3.8 & 3.7 \\
\hline \multicolumn{3}{|l|}{ Indicateur de précarité } \\
\hline Score ÉPICES $(m, \sigma)$ & $44.09(25.03)$ & $40.93(24.53)$ \\
\hline « Précaires » $(\%)$ & 50 & 51.5 \\
\hline « Non précaires » $(\%)$ & 50 & 48.5 \\
\hline \multicolumn{3}{|l|}{ Perspective Temporelle $(m, \sigma)$} \\
\hline Passé-positif & $3.24(0.68)$ & $3.37(0.68)$ \\
\hline Passé-négatif & $2.64(0.92)$ & $2.75(0.97)$ \\
\hline Présent-fataliste & $2.53(.92)$ & $2.51(0.86)$ \\
\hline Présent-hédoniste & $3.13(1.00)$ & $2.85(0.96)^{*}$ \\
\hline Futur & $3.40(0.75)$ & $3.41(0.72)$ \\
\hline \multicolumn{3}{|l|}{$H A D(m, \sigma)$} \\
\hline HAD-Anxiété & $7.96(3.53)$ & $9.17(4.07)^{* *}$ \\
\hline HAD-Dépression & $5.36(3.44)$ & $5.09(3.23)$ \\
\hline
\end{tabular}

$* p \leq .05 .{ }^{* *} p \leq .01 . * * * p \leq .001$.

des différences importantes entre les sexes concernant les CSP chez les salariés (établies à partir des professions déclarées réparties en trois catégories : supérieure: chefs d'entreprise, cadres et professions intellectuelles supérieures ; intermédiaires : Professions intermédiaires ; inférieures: Employés, ouvriers), les femmes étant moins nombreuses dans les catégories supérieures, et plus nombreuses dans les catégories intermédiaires ou inférieures. Concernant les autres variables, on constate un effet du sexe sur la dimension présent hédoniste $(\mathrm{PH})$ de la PT, les hommes étant plus orientés vers cette dimension que les femmes, ainsi qu'un effet sur le score d'anxiété qui apparaît supérieur chez les femmes. Ces observations rejoignent celles généralement rapportées concernant les différences liées au sexe par rapport à ces deux construits.

\section{Insertions sociales et PT}

Afin d'étudier les variations de la PT en fonction des différentes caractéristiques des insertions sociales, nous avons réalisé des ANOVA sur chaque souséchelle de la ZTPI à partir des données sociodémographiques recueillies. Les résultats de ces analyses sont récapitulés dans le tableau 2. On constate en premier lieu que le niveau de diplôme introduit des différences au niveau des registres passé négatif $(\mathrm{PN})$, présent fataliste $(\mathrm{PF})$ et présent hédoniste $(\mathrm{PH})$. Ainsi, les sujets sans diplôme ou ayant un diplôme inférieur au bac se situent plus dans les registres $\mathrm{PN}, \mathrm{PF}$ et $\mathrm{PH}$ que les autres. La situation vis-àvis de l'emploi introduit également une différence au niveau du registre passé négatif, les personnes au chômage étant plus orientées vers ce registre que les sujets ayant un emploi. Chez les salariés, la CSP introduit elle aussi des différences sur le registre $\mathrm{PN}$, vers lequel sont plus orientées les catégories inférieures et intermédiaires. En revanche, les catégories supérieures apparaissent plus orientées vers le futur que les catégories inférieures.

Enfin, la dimension relationnelle a elle aussi un effet sur certains registres de la PT. Ainsi, les sujets déclarant ne pas avoir de relation avec leur famille se situent plus dans le passé négatif et moins dans le futur que les sujets déclarant entretenir des liens avec leur famille. Le même effet apparaît concernant les relations conjugales, les sujets déclarant avoir une relation se situant moins dans le passé négatif que ceux déclarant ne pas en avoir. Par ailleurs, on constate que le type de situation par rapport au logement (propriétaire, locataire, hébergé) n'introduit pas de différences dans la PT.

\section{Niveau de précarité et $P T$}

Lorsque l'on cherche à prédire la PT à partir du niveau de précarité mesuré par le score ÉPICES, les analyses de régressions multiples (contrôlées pour 
Tableau 2 : Variations sur les cinq sous-échelles de la ZTPI en fonction des caractéristiques sociodémographiques (ANOVA)

\begin{tabular}{|c|c|c|c|c|c|}
\hline \multirow[b]{2}{*}{ Variables } & \multicolumn{5}{|c|}{ Sous-échelles de la ZTPI } \\
\hline & Passé Négatif & Passé Positif & Présent Fataliste & Présent Hédoniste & Futur \\
\hline \multicolumn{6}{|c|}{ Niveau de diplôme $(m, \sigma)$} \\
\hline Aucun & $2.81(1.02)$ & $3.02(0.90)$ & $2.50(0.89)$ & $2.68(0.98)$ & $3.26(0.93)$ \\
\hline Inf. bac & $2.98(0.99)$ & $3.32(0.76)$ & $2.90(0.82)$ & $3.14(1.03)$ & $3.42(0.72)$ \\
\hline $\mathrm{Bac}$ & $2.69(0.89)$ & $3.47(0.57)$ & $2.50(0.84)$ & $2.70(0.82)$ & $3.45(0.70)$ \\
\hline Sup. bac & $2.43(0.91)$ & $3.28(0.63)$ & $2.27(0.82)$ & $3.03(0.99)$ & $3.47(0.67)$ \\
\hline$F(3$ 214) & $4.38 * *$ & $2.05 ; n s$ & $7.16 * * *$ & $2.48 *$ & $0.56 ; n s$ \\
\hline \multicolumn{6}{|l|}{ Activité $(m, \sigma)$} \\
\hline 'Travail & $2.58(0.94)$ & $3.30(0.64)$ & $2.43(0.85)$ & $2.88(1.13)$ & $3.44(0.67)$ \\
\hline Chômage & $2.88(0.95)$ & $3.17(0.71)$ & $2.60(0.92)$ & $3.03(0.90)$ & $3.33(0.76)$ \\
\hline$F(1,186)$ & $4.77 *$ & $1.59 ; n s$ & $1.78 ; n s$ & $0.97 ; n s$ & $1.15 ;$ ns \\
\hline \multicolumn{6}{|l|}{$\operatorname{CSP}(m, \sigma)$} \\
\hline Prof. Sup. & $2.08(0.67)$ & $3.20(0.57)$ & $2.23(0.65)$ & $3.06(1.03)$ & $3.62(0.57)$ \\
\hline Prof. Int. & $2.73(0.91)$ & $3.34(0.64)$ & $2.30(0.96)$ & $2.95(1.26)$ & $3.40(0.72)$ \\
\hline Prof. Inf. & $2.76(0.97)$ & $3.36(0.65)$ & $2.61(0.89)$ & $2.61(1.07)$ & $3.25(0.66)$ \\
\hline$F(2,81)$ & $5.36 * *$ & $0.48 ; n s$ & $1.62 ; n s$ & $1.15 ; n s$ & $2.15 *$ \\
\hline \multicolumn{6}{|c|}{ Relations familiales $(m, \sigma)$} \\
\hline Oui & $2.62(0.94)$ & $3.31(0.69)$ & $2.52(0.86)$ & $2.98(1.00)$ & $3.45(0.71)$ \\
\hline Non & $3.01(0.91)$ & $3.08(0.63)$ & $2.27(0.76)$ & $2.85(0.98)$ & $3.16(0.84)$ \\
\hline$F(1,228)$ & $5.74 * *$ & $3.26 ; n s$ & $2.36 ; n s$ & $0.47 ; n s$ & $4.33 *$ \\
\hline \multicolumn{6}{|c|}{ Relation conjugale $(m, \sigma)$} \\
\hline Oui & $2.53(0.96)$ & $3.36(0.66)$ & $2.43(0.78)$ & $2.99(1.04)$ & $3.46(0.68)$ \\
\hline Non & $2.86(0.93)$ & $3.20(0.72)$ & $2.60(0.95)$ & $2.96(0.97)$ & $3.36(.079)$ \\
\hline$F(1,234)$ & $6.84 * *$ & $3.00 ; n s$ & $2.34 ; n s$ & $0.05 ; n s$ & 1.17 ; ns \\
\hline
\end{tabular}

$$
* p \leq .05 . * * p \leq .01 . * * * p \leq .001
$$

l'âge et le sexe) font apparaître un effet significatif pour les registres passés et le registre futur. Ainsi, plus les sujets ont un niveau de précarité important, plus ils sont centrés sur un passé négatif $(\beta=.25, t$ $=4.28, p<.001)$ et moins sur un passé positif $(\beta=$ $-.12, t=-2.00, p<.05)$. Le niveau de précarité est par ailleurs négativement lié à l'orientation vers le futur $(\beta=-.12, t=-1.92, p=.05)$, suggérant que plus le niveau de précarité augmente moins les individus se centrent sur le futur.

\section{Niveau de précarité et anxiété et dépression}

Les liens dans notre échantillon entre le niveau de précarité et les deux scores de l'échelle $H A D$ (HADA, HAD-D) ont été explorés au travers d'analyses de régression multiples (contrôlées pour l'âge et le sexe), afin de prédire les réponses à ces indicateurs de troubles psychologiques à partir du score de précarité. Le niveau de précarité apparaît positivement lié à l'anxiété et à la dépression, suggérant une augmentation du niveau d'anxiété $(\beta=.15, t=2.60, p$ $<.01)$ et de dépression $(\beta=.28, t=4.88, p<.001)$ à mesure que le score de précarité augmente. Cet effet peut être aussi illustré par les ANOva réalisées pour comparer les deux groupes, «précaires » et «non précaires »: chez les premiers, les scores sur les deux sous-échelles de la HAD sont significativement plus importants que chez les seconds (HAD$\mathrm{A}: m 1=9.15, \sigma=4.19, m 2=8, \sigma=3.39, F(1,274)$ $=6.204, p=.01 ;$ HAD-D : $m 1=6.05, \sigma=3.38, m 2$ $=4.37, \sigma=3.06, F(1,274)=18.381, p<.001)$.

\section{PT et anxiété et dépression}

Afin d'étudier les liens entre la PT et les troubles psychologiques, nous avons réalisé une série de régressions multiples (contrôlées pour l'âge et le sexe) afin de prédire les scores aux deux sous-échelles de la HAD à partir des scores aux différentes sous- 
Tableau 3 : Prédictions des scores aux deux sous-échelles de la HAD à partir des scores aux cinq sous-échelles de la ZTPI : régressions multiples (coefficients standardisés)

\begin{tabular}{ccccccc}
\hline & \multicolumn{4}{c}{ Sous-échelles de la ZTPI } & \multicolumn{2}{c}{ Résumé du modèle } \\
Sous-échelles HAD & PP & PN & PF & PH & F & $R^{2}=.15, F(7,267):=6.84^{* * *}$ \\
HAD-Anxiété & -.02 & $.35 * * *$ & .01 & .04 & -.01 & $R^{2}=.10, F(7,267):=4.07 * * *$ \\
HAD-Dépression & -.01 & $.27 * * *$ & .06 & -.03 & -.03 & \\
\hline
\end{tabular}

$* p \leq .05 . * * \leq .01 . * * * p \leq .001$.

Tableau 4 : Modèles de prédiction des scores aux deux sous-échelles de HAD. Régressions multiples

\begin{tabular}{|c|c|c|c|c|}
\hline & \multicolumn{2}{|c|}{ HAD-Anxiété } & \multicolumn{2}{|c|}{ HAD-Dépression } \\
\hline Modèle 1 & $B$ & $t$ & $B$ & $t$ \\
\hline Age & -.06 & -1.07 & .07 & 1.27 \\
\hline Sexe & .16 & $2.71 * *$ & -.01 & -.28 \\
\hline Score précarité & .15 & $2.60 * *$ & .28 & $4.88 * * *$ \\
\hline Résumé du modèle & \multicolumn{2}{|c|}{$R^{2}=.05, F(3,271):=4.95 * *$} & \multicolumn{2}{|c|}{$R^{2}=.09, F(3,271):=8.70 * * *$} \\
\hline Modèle 2 & $B$ & $t$ & $B$ & $t$ \\
\hline Age & -.03 & -.57 & .09 & 1.67 \\
\hline Sexe & -.14 & $2.46^{* * *}$ & -.03 & -.56 \\
\hline Score précarité & .07 & 1.22 & .22 & $3.85^{* * *}$ \\
\hline $\mathrm{PN}$ & .33 & $5.71 * * *$ & .23 & $3.97 * * *$ \\
\hline Résumé du modèle & \multicolumn{2}{|c|}{$R^{2}=.15, F(4,270):=12.30 * * *$} & \multicolumn{2}{|c|}{$R^{2}=.14, F(4,270):=10.84^{* * *}$} \\
\hline
\end{tabular}

$* p \leq .05 . * * p .01 . * * * p \leq .001$.

échelles de la ZTPI. Les résultats des analyses sont récapitulés dans le tableau 3 (cf.ci-dessus). Seul le registre passé négatif se révèle lié aux indicateurs d'anxiété et de dépression, les personnes plus orientées vers ce registre ayant des scores plus élevés dans les deux cas.

\section{Conclusions intermédiaires}

Ces premières analyses mettent en avant un certain nombre de constats. Premièrement, on retrouve dans cet échantillon les constats répétés des enquêtes à l'échelle nationale concernant le lien entre précarité et troubles psychologiques (e.g. La Rosa et al., 2000). Le niveau de précarité apparaît ainsi prédictif des scores d'anxiété et de dépression mesurés par l'échelle HAD. Deuxièmement, les résultats mettent en évidence les variations dont la PT fait l'objet en fonction des insertions sociales, et notamment la dimension passé négatif qui apparaît particulièrement liée aux insertions sociales les plus défavorisées. Celle-ci est plus saillante chez les personnes à faible niveau de diplôme, chez celles qui sont au chômage, qui appartiennent aux CSP inférieures lorsqu'elles travaillent, qui n'ont pas de relations avec leur famille ou ne vivent pas en couple. D'autre part, le niveau de diplôme apparaît comme un facteur important par rapport à plusieurs registres de la PT. Enfin, le registre futur de la PT apparaît peu lié aux insertions sociales. Par ailleurs, le niveau de précarité, mesuré par le score multidimensionnel ÉPICES est lié aux registres passés et futur de la PT. C'est dans le cas du registre passé négatif que le lien avec cet indicateur de précarité apparaît le plus significatif. Ce constat rejoint les travaux qui soulignent l'importance à accorder à la "fragilisation biographique » dans l'analyse des situations de précarité (Bouchayer et Verger, 1994). Il amène également à souligner la nécessité de considérer non seulement l'orientation temporelle, mais également l'attitude négative ou positive qui y est attachée, la dimension passé positif ne faisant pas l'objet des mêmes variations.

Troisièmement, le registre passé négatif de la PT apparaît significativement lié aux niveaux d'anxiété et de dépression, ce qui rejoint le constat établi dans la littérature concernant la relation entre "rumination négative » du passé et souffrance psychologique (Holman et Silver, 1998). En revanche, nos résultats ne rejoignent pas complètement les observations de Zimbardo et Boyd (1999), selon lesquelles toutes les dimensions de la PT apparaissent significativement liées aux indicateurs d'anxiété ou 
de dépression ${ }^{1}$. Par ailleurs, il faut noter que le score d'anxiété apparaît plus fortement liée au registre PN de la PT qu'au niveau de précarité, ce qui n'est pas le cas pour la dépression. Ce constat souligne d'une part le rôle prépondérant de ce registre dans l'émergence de troubles anxieux, et d'autre part suggère la nécessité de distinguer les deux dimensions mesurées par la HAD.

L'ensemble de ces premiers résultats permet de conclure à l'intérêt de la PT comme variable psychosociale dans l'analyse des situations de précarité. Plus particulièrement, ces résultats invitent à considérer l'importance du registre passé négatif de la PT (PT-PN) et à cibler l'analyse sur son rôle dans les liens observés entre niveau de précarité et troubles psychologiques. L'hypothèse qui fonde la suite des analyses est donc celle de l'intervention de ce registre comme vecteur de vulnérabilisation par rapport aux troubles anxieux et dépressifs. En d'autres termes, nous faisons l'hypothèse que le passé négatif agit comme une variable médiatrice dans le lien entre niveau de précarité et niveaux d'anxiété et de dépression.

\section{Précarité, passé négatif et anxiété et dépression}

Afin de statuer sur le rôle joué par la PT-PN, nous avons utilisé une série de modèles de régression ( $f$. Holmbeck, 1997). Cette procédure permet de tester l'hypothèse d'un effet médiateur de la PT-PN dans le lien entre précarité et anxiété d'une part et précarité et dépression d'autre part. L'existence d'un effet médiateur est établie au niveau des analyses de régression si plusieurs effets sont vérifiés (cf. Baron et Kenny, 1986) : 1) La significativité de la relation entre le prédicteur et la variable dépendante; 2) La significativité de la relation entre le prédicteur et la variable médiatrice ; 3) La diminution significative du lien entre le prédicteur et la variable dépendante lorsque la variable médiatrice est ajoutée au modèle ; 4) La significativité de l'effet indirect. Pour les deux variables dépendantes (HAD-A et HAD-D), les résultats des étapes 1 et 3 sont présentés dans le tableau 4 (cf.ci-contre), la relation entre le prédicteur (le score ÉPICES) et la variable modératrice (PN-PT) ayant déjà été présentée $(\beta=.25, t=4.28, p<.001$; âge et sexe contrôlés).

La figure 1 illustre les résultats obtenus. Ces résultats confirment l'hypothèse selon laquelle le registre
Figure 1 : Effets médiateurs du registre Passé Négatif de la PT sur l'anxiété (1.a): et sur la dépression (1.b).
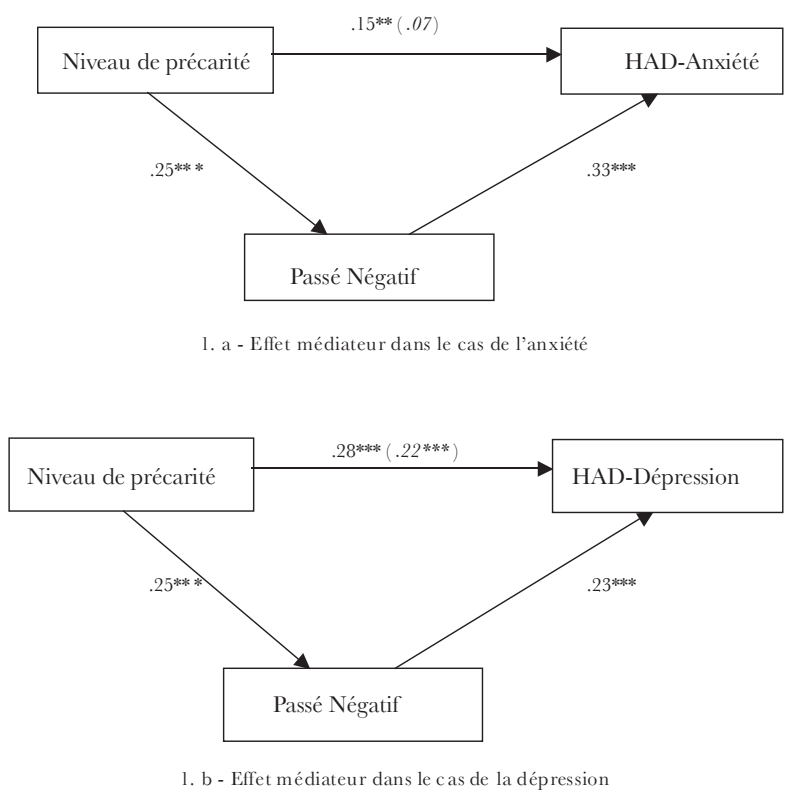

Note : Le coefficient de régression entre parenthèses et en italiques correspond à l'effet direct du prédicteur sur la variable dépendante une fois la variable médiatrice introduite $;{ }^{*} \mathrm{p} \leq .05 . * * \mathrm{p} \leq .01 . * * * \mathrm{p} \leq .001$.

passé négatif de la PT constitue une variable médiatrice dans le lien entre niveau de précarité et souffrance psychologique. Ainsi, la relation entre le niveau de précarité et les scores aux deux indicateurs HAD-A et HAD-D est affaiblie lorsque la variable PT-PN est intégrée à l'équation de régression, et ce de manière plus importante dans le cas de l'anxiété que dans le cas de la dépression. En appliquant le test de Sobel (Sobel, 1982), on constate pourtant que cet effet de médiation (effet indirect) est significatif à la fois pour l'anxiété $(z=3.54, p=.0003)$ et pour la dépression $(z=2.98, p=.002)$. Le registre PT-PN apparaît donc comme une variable explicative susceptible de rendre compte (au moins partiellement) du mécanisme par lequel la relation entre niveau de précarité et scores aux deux dimensions de la HAD s'établit.

Il faut également souligner le fait que cet effet médiateur apparaît différencié selon la dimension considérée, anxiété ou dépression. En effet, la prise en compte du registre PN rend non significatif l'effet du niveau de précarité dans le cas de l'anxiété et

1. Ce constat pourrait s'expliquer par différents facteurs : échantillons (étudiants dans la recherche de Zimbardo et Boyd); indicateurs mesurant les troubles psychologiques ; version réduite de la ZTPI dans la présente étude ; contexte socioculturel différent ; ... Il est difficile de statuer sur ces différences dans le cadre du présent travail. 
non pas dans le cas de la dépression, où le niveau de précarité reste un prédicteur significatif. Bien que la diminution de l'effet direct du niveau de précarité soit équivalente dans les deux cas, ce résultat suggère qu'anxiété et dépression ne sont pas des construits assimilables, et répondent à des logiques spécifiques.

\section{Discussion}

En lien avec nos objectifs, plusieurs constats principaux émergent à l'issue de cette étude. Premièrement, les résultats présentés démontrent les variations dont la P'T fait l'objet en fonction de différentes dimensions des insertions sociales (capital culturel, insertion professionnelle, capital relationnel). Ce résultat, particulièrement significatif dans le cas des registres passés (positif et négatif), amène à souligner à nouveau la dimension socialement régulée de la PT et les limites d'une approche centrée uniquement sur les différences interindividuelles. Ces résultats illustrent également l'impact que peut avoir le niveau de précarité sur la PT, offrant ainsi un appui empirique à l'hypothèse d'un rapport au temps marqué par les conditions d'existence précaires (Paugam, 2000). Cet effet concerne essentiellement le registre passé négatif, corroborant ainsi les analyses des liens entre parcours biographiques et vécus des situations de précarité (Billiard et al., 2000). Il est à cet égard intéressant de noter que l'effet inverse n'apparaît pas concernant le registre passé positif, mettant en évidence la nécessité de considérer ces deux registres de manière distincte. Deuxièmement, les résultats rejoignent les constats récurrents des recherches socio-épidémiologiques, et confirment que les insertions sociales précaires sont associées à différents indices de souffrance psychologique (Kovess-Masféty, 2001). De plus, le registre passé négatif de la PT apparaît comme un facteur en lien avec les niveaux observés d'anxiété et de dépression, suggérant les conséquences que peut avoir un rapport au passé marqué par la négativité sur les troubles psychologiques.

\section{L'effet médiateur de la PT}

Troisièmement, et conformément à notre hypothèse médiationnelle, la PT apparait comme une variable psychosociale importante et utile pour éclairer la complexité des liens observés entre précarité et souffrance psychologique. Ainsi, l'effet médiateur du registre passé négatif montre que l'impact de l'insertion sociale précaire sur la souffrance psychologique semble s'établir au travers de l'élaboration d'un rapport négatif au passé. En s'appuyant sur les relations causales établies dans la littérature (e.g. Holman et Silver, 1998), et en considérant les limites inhérentes aux données transversales sur lesquelles s'appuie cette étude, on peut suggérer une interprétation de l'effet médiateur de la PT. On pourrait postuler que la précarité a un impact sur les troubles psychologiques, et en particulier sur l'anxiété, en partie parce qu'elle agit sur ou par son action sur le registre passé négatif de la PT. Conformément au rôle attribué aux variables médiatrices, la PT-PN peut ainsi participer à expliquer comment les évènements ou les situations prennent leur signification subjective (Baron et Kenny, 1986). Cependant, il faut souligner que ce mécanisme ne peut à lui seul expliquer l'ensemble des troubles psychologiques liés aux situations de précarité, ceux-ci ne restant que partiellement expliqués par les modèles testés (cf. pourcentages de variance expliquée). Ces résultats suggèrent néanmoins que pour comprendre comment la précarisation altère le rapport à soi, il est primordial d'analyser le rôle joué par le temps psychologique. Ils soulignent également combien les processus de précarisation, loin d'être spécifiables seulement au travers d'indicateurs objectifs, doivent aussi être analysés en fonction du rapport que les individus entretiennent à leur situation, et plus particulièrement à leur trajectoire.

Pour autant, la prise en compte de la PT dans les liens entre précarité et troubles psychologiques ne réduit pas leur complexité. En particulier concernant la distinction qui s'établit entre anxiété et dépression par rapport au rôle médiateur du registre passé négatif. La perte de significativité de l'effet direct du niveau de précarité spécifiquement pour l'anxiété constitue à cet égard un constat particulièrement important. Dans ce cas, il apparaît que l'effet du niveau de précarité se trouve presque complètement médiatisé par la PT-PN, contrairement à la dépression où cet effet reste significatif. Il semble ainsi que l'anxiété, qui représente une expérience subjective d'insécurité et de vulnérabilité, marquée avant tout par la peur, soit liée aux situations de précarité par le biais du rapport que les individus entretiennent à leur passé. Rapport qui peut refléter une « fragilisation biographique » rétrospective, vecteur d'un sentiment de vulnérabilité lié à une insertion sociale précaire. En revanche, le registre PN joue un rôle moins important dans le cas de la dépression, qui représente un état de ralentissement et de troubles de l'humeur liés à des affects négatifs comme l'insatisfaction, la dévalorisation, le pessimisme. Le lien que cette douleur morale, principalement marquée par la tristesse, entretient avec le niveau de préca- 
rité ne semble que partiellement médiatisé par ce registre. Ces résultats confirment que les construits « anxiété » et « dépression » tels qu'ils sont mesurés par la HAD, quoique très liés entre eux, ne sont pas assimilables (cf. Bjelland et al., 2002) et semblent répondre à des logiques distinctes.

Cet effet différentiel peut apparaître comme contreintuitif lorsque l'on considère la dimension des affects. En effet, le registre PN mesuré par la ZTPI se caractérise par des affects négatifs liés au passé, ce qui pourrait amener à centrer sur cette composante affective l'interprétation du rôle joué spécifiquement par ce registre. Les résultats obtenus, montrant un rôle moins important du passé négatif dans le cas de la dépression, amènent à privilégier une autre piste d'interprétation. En effet, les résultats concernant l'anxiété suggèrent que l'effet médiateur du registre passé négatif agit surtout dans le sens d'une incertitude anxiogène vis-à-vis de la situation présente. Il semble ainsi que la perception insécurisante de l'insertion sociale précaire et la vulnérabilité psychologique qu'elle peut engendrer, dont l'anxiété représente un indicateur, reposent sur un mécanisme de fragilisation porté par la présence rétrospective et la rumination d'un passé négatif. Dans ce cadre, ces résultats rejoignent les analyses sociologiques qui situent la spécificité des insertions sociales précaires dans la déstabilisation et l'insécurité, objective et subjective, qu'elles établissent en rapport aux trajectoires (Castel, 2003). De plus, ils les complètent en soulignant le rôle joué par le rapport subjectif et socialement régulé que les individus entretiennent à leur passé. Par ailleurs, ces résultats suggèrent que le vécu des situations de précarité, et plus particulièrement le rapport à soi que ce vécu implique, se trouve sous l'influence du passé psychologique et des affects qui le marquent. Comme le soulignait déjà Lewin (1942), ce ne sont pas seulement les épreuves et les privations en elles-mêmes qui déterminent le niveau de souffrance des individus mais également certains aspects de leur perspective temporelle, qui donnent à ces expériences leur sens psychologique. En conclusion, ces résultats conduisent à souligner qu'il est primordial d'analyser le rôle joué par le temps psychologique comme construit psychosocial participant à constituer la situation totale présente et à donner aux insertions sociales leur signification subjective. Cette prise en compte peut ainsi permettre de mieux comprendre la façon dont la précarisation altère le rapport à soi.

\section{Limites de l'étude}

Pour considérer la portée des résultats présentés, il faut prendre en compte les principales limites inhérentes à cette étude.

Tout d'abord, la principale limitation provient du caractère transversal des données recueillies, qui ne permet pas de statuer sur la nature causale des liens observés. L'hypothèse de médiation, se fondant sur le postulat de relations causales (Baron et Kenny, 1986), ne saurait ainsi être validée par ces données transversales. Néanmoins, différents éléments amènent à considérer ces résultats comme significatifs et intéressants pour tracer certaines pistes de travail pour des recherches futures. D'une part, les liens de causalité postulés dans la présente étude sont cohérents avec les propositions théoriques dans le champ considéré (Fahoda, 1982 ; Bonizeell et Zimbardo, 2004), ainsi qu'avec les résultats déjà établis au travers de recherches longitudinales (lien causal entre rapport au temps et troubles psychologiques, e.g. Waters et Muller, 2003 ; et entre insertions sociales et rapport au temps; e.g. Wanberg et Griffiths, 1997 ; rôle médiateur de la PT, e.g. Holman et Silver, 1998). D'autre part, la nature des variables étudiées (situation sociale, variable sociocognitive générale et état psychique) correspondent de manière "typique » aux relations étudiées dans le cadre d'analyses médiationnelles (cf. Frazier et al., 2004). Ces éléments offrent ainsi un appui non négligeable aux interprétations proposées, qui par ailleurs sont moins fondées sur le postulat d'une causalité simple que sur celui de l'interdépendance circulaire qui s'établit entre les individus et leur environnement. Il apparaît par ailleurs nécessaire de tenir compte pour des recherches futures de la possibilité d'effets de feedback par lesquels, par exemple, la souffrance psychologique peut alimenter la dégradation des conditions de vie par l'évitement et le retrait qu'elle peut engendrer (Jahoda, 1992). De même, l'analyse médiationnelle qui place la PT en variable intermédiaire, ne doit pas faire oublier les relations réciproques qui peuvent s'établir entre rapport au temps et souffrance psychologique. Cette dernière peut en effet elle-même entraîner des "replis temporels stratégiques », se traduisant par exemple par un surinvestissement du passé dans le rapport que les individus construisent à leur trajectoire (BarusMichel, 1990). Ces limitations soulignent la nécessité de recherches longitudinales et-ou expérimentales, afin de confirmer et compléter les observations rapportées ici. De telles études permettront de statuer sur la dynamique des processus à l'œuvre dans les rapports complexes entre insertions sociales, PT et santé psychologique. 
Ensuite, il faut considérer les limites liées aux indicateurs utilisés. Concernant l'outil de mesure de la PT, une première limite provient de la version réduite utilisée, et de sa qualité psychométrique pour mesurer le construit visé (niveau des indices de consistance, déséquilibre du nombre des items dans chaque dimension). Cet élément pourrait en partie expliquer les différences observées dans les résultats par rapport à certaines données de la littérature (e.g. liens entre troubles psychologiques et registre présent fataliste de la PT; cf. Zimbardo et Boyd, 1999). Une seconde limite de cet outil tient au fait que le rapport au temps ne saurait être entièrement décrit par la ZTPI. Celle-ci ne mesure en effet que certaines composantes de la PT (orientation et attitude), qui ne représente elle-même qu'un aspect du temps psychologique. Par ailleurs, les faibles liens établis concernant le registre futur peuvent tenir en partie à l'unidimensionnalité de cette dimension dans l'échelle, centrée uniquement sur la planification et l'accomplissement des buts. La décomposition de ce construit en relation aux attitudes qui peuvent l'accompagner (par exemple : pessimisme, optimisme) représente à cet égard un objectif de travail incontournable. Malgré ces limites, les résultats présentés confirment l'intérêt de l'échelle ZTPI comme outil multidimensionnel de mesure de la PT, et sa pertinence pour étudier le rôle complexe joué par cette variable dans les rapports que les individus entretiennent à leur environnement (Zimbardo et Boyd, 1999). Concernant l'indicateur de précarité utilisé, il ne saurait mesurer de manière exhaustive les insertions sociales précaires. Néanmoins, le score ÉPICES se trouve être à l'heure actuelle un des seuls indicateurs multidimensionnels de mesure du niveau de précarité des situations individuelles. Par sa nature continue, cet indicateur évite que l'ensemble des analyses ne repose que sur une classification, forcément arbitraire, entre «précaires » et «non précaires ». Ces éléments en font un outil pertinent dans les recherches concernant les phénomènes de précarisation et leurs effets sur la santé. Concernant l'outil utilisé pour mesurer les troubles psychologiques, bien que largement utilisé et validé, il ne permet qu'une évaluation générale de l'état anxio-dépressif. La souffrance psychologique que la HAD permet d'évaluer représente un indicateur utile, mais insuffisant, de l'impact de l'expérience des situations de précarité sur le rapport à soi. Enfin, certaines limites liées à l'échantillonnage ne permettent pas de généraliser sans précaution les résultats obtenus ${ }^{2}$.
Malgré ces limites, ces résultats ouvrent des perspectives dans différentes directions. D'une part, les régulations sociales dont la PT fait l'objet invitent à considérer de façon plus approfondie la nature psychosociale de cette variable. Comme l'a souligné de manière princeps Lewin (1942), celle-ci s'ancre dans les rapports d'interdépendance dynamique qu'entretiennent les individus et les groupes à leur environnement. En ce sens, l'étude de la PT réclame, comme pour d'autres variables de personnalité, un travail de contextualisation afin d'analyser comment le rapport au temps est situé dans- et actualisé par- les rapports sociaux et les places sociales qu'ils définissent. Dans ce cadre, la nécessité de prendre en compte la réciprocité de perspectives entre réalité psychologique et réalité sociale se pose avec d'autant plus de force dans l'étude psychosociale du rapport au temps. Cette étude souligne également l'importance du registre passé de la PT et son caractère socialement régulé, aspect trop souvent négligé et qui pourtant semble être incontournable dans l'analyse des phénomènes de précarisation et de leurs conséquences. D'autre part, cette étude offre des hypothèses de travail intéressantes dans le cadre de l'analyse des déterminants sociaux de la santé et dans l'étude psychosociale des insertions spécifiques que constituent les situations de précarité. Enfin, ces résultats ouvrent des perspectives utiles dans le domaine des applications psychosociales, en soulignant l'importance de considérer le rapport au temps, et plus particulièrement la « fragilité biographique » dans le champ de l'accompagnement des populations en situation de précarité.

2. Parmi lesquelles on peut noter : la limitation à une population de 25 à 44 ans ; la spécificité du lieu de recrutement qui exclut de facto les populations en très grande précarité ; la période à laquelle s'est déroulée l'enquête. 


\section{RÉFÉRENCES}

Allport G. W. (1948): Foreword. In K. Lewin, Resolving social conflicts. New York, Harper et Brothers, pp. 7-14.

- Apostolidis T. et Fieulaine N. (2004): Validation française de l'échelle de temporalité The Zimbardo Time Perspective Inventory. Revue Européenne de Psychologie Appliquée, Vol. 54, № 3, pp. 207-217.

- Appay B. (1997): Précarisation sociale et restructurations productives. In B. Appay et A. Thébaud-Mony (Dirs.), Précarisation sociale, travail et santé. Paris, IRESCO, pp. 509-553

- Baron R. M. et Kenny D. A. (1986): The moderator-mediator variable distinction in social psychological research: Conceptual, strategic, and statistical considerations. Fournal of Personality and Social Psychology, Vol. 51, № 6, pp. 1173-1182.

- Barus-Muchel J. (1990): Le temps et la distance dans les relations sociales. Temporalistes, 13, pp. 9-15.

- Bihan H., Laurent S., Sass G., Nguyen G., Huot G., Moulin J. J., Guegen R., LeToulemin P., LeClésiau H., LaRosa E., Reach G. et Cohen R. (2005): Association among individual deprivation, glycemic control, and diabetes complications. The EPICES score. Diabetes Care, Vol. 28, № 11, pp. 2680-2685.

- Billiard I., Debordeaux D. et Lurol M. (2000): Vivre la précarité, trajectoires et projets de vie. La Tour d'Aigues, Éditions de l'Aube.

- BJelland I., Dahl A. A., Haug T. T. et Neckelmann D. (2002): The validity of the Hospital Anxiety and Depression Scale: An updated literature review. Fournal of Psychosomatic Research, Vol. 52, No 2, pp. 69-77.

- Boniwell I. et Zimbardo P. G. (2004): Balancing one's time perspective in pursuit of optimal functioning. In P. A. Linley et S. Joseph (Dirs.), Positive psychology in practice. Hoboken, Wiley, pp. 165-178.

- Bouchayer F. et Verger D. (1994): Trajectoires sociales et inégalités. Ramonville Saint-Agne, Érès.

Castel R. (2003): Linsécurité sociale. Qu'est-ce qu'être protégé? Paris, Seuil.

Cazals-Ferré M. P. et Llorca M. C. (2002): L'impact de la précarité sur la vulnérabilité et la santé des individus. Pratiques Psychologiques, 4 , pp. 51-64.

- D'Alessio M., Guarino A., DePascalis V. et Zimbardo P. G. (2003): Testing Zimbardo's Stanford Time Perspective Inventory (STPI): Short Form. An Italian study. Time et Society, Vol. 12, No 2/3, pp. 333347.

- Delors F. et Hubert M. (2000): Revisiting the concept of « vulnerability ». Social Science et Medicine, Vol. 50, N 11, pp. 1557-1570.

- Epel S. E., Bandura A. et Zimbardo P. G. (1999): Escaping homelessness: The influences of self-efficacy and time perspective on coping with homelessness. Fournal of Applied Social Psychology, Vol. 29, ํ3, pp. 575-596.

- European Foundation for the Improvement of Living AND Working Conditions (1997): Precarious employment and working conditions in the European Union. Dublin, Author.

- Feather N. T. (1997): Economic deprivation and the psychological impact of unemployment. Australian Psychologist, Vol. 32, $\mathrm{N}^{\circ}$ 1, pp. 3745.

- Fingerman K. L. et Perlumutter M. (2001): Future time perspective and life events across adulthood. The fournal of General Psychology, Vol. $122, \mathrm{~N}^{\circ} 1$, pp. 95-111.

- Frazier P. A., Tix A. P. et Barron K. E. (2004): Testing moderator and mediator effects in counseling psychology research. Fournal of Counseling Psychology, Vol. 51, N, 1, pp. 115-134.

- GuÉguen R., SAss G. et ÉPICES Working Group (2005): The ÉPICES score: an individual index of material and social deprivation related to health status. Part I - Setting up the score. Document disponible en ligne: http://www.cetaf.asso.fr/protocoles/precarite/protocoles_epices.htm Dernière consultation le 9 mai 2006.
- Holman E. A. et Silver C. S. (1998): Getting «stuck» in the past: Temporal orientation and coping with trauma. Fournal of Personality and Social Psychology, Vol. 74, No 5, pp. 1146-1163.

- Holmbeck G. N. (1997): Toward terminological, conceptual, and statistical clarity in the study of mediators and moderators: Examples from the child-clinical and psychology literatures. Fournal of Counseling and Clinical Psychology, Vol. 65, No 4, pp. 399-410.

- JaHoda M. (1982): Employment and unemployment. Cambridge, University Press.

-Jahoda M. (1992): Reflections on Marienthal and after. Fournal of Occupational and Organizational Psychology, Vol. 65, N 1, pp. 355-358.

- Joubert M., Chauvin P. et Facy F. (2001): Précarisation, risque et santé. Paris, Inserm.

- Kovess-Masféty V. (2001): Précarité et santé mentale. Rueil-Malmaison, Doin.

- Krauss H. H. (1967): Anxiety and temporal perspective. Fournal of Clinical Psychology, Vol. 23, $\mathrm{N}^{\circ}$ 3, pp. 340-342.

- La Rosa L. (1998): Santé, précarité et exclusion, Paris, PUF.

- La Rosa E., Consoli S. M., Le Cilesiau H., Birouste J., Joubert M. et Soufi K. (2000): Étude de la souffrance psychosociale et de ses facteurs modérateurs chez les consultants en situation de précarité d'un centre de prévention sanitaire et sociale. Revue d'Épidémiologie et de Santé Publique, Vol. 48, № 4, pp. 351-362.

Lamm H., Schmidt R. W. et Trommsdorf G. (1976): Sex and social class as determinants of future orientation in adolescents. Fournal of Personality and Social Psychology, Vol. 34, pp. 317-326.

- Lebas J. et Ghauvin P. (1998): Précarité et santé. Paris, Flammarion.

- LÉPINE J.-P. (1993): Étude épidémiologique des troubles dépressifs et anxieux dans la population générale. Annales Médico-Psychologiques, Vol. 151, pp. 618-623.

- Lewin K. (1942): Time Perspective and Morale. In G. Watson (Dir.), Civilian Morale. Boston, Houghton Mifflin, pp. 48-70.

- Lewin K. (1951): Field theory in social sciences. New York, Harper.

- Paugam S. (2000): Le salarié de la précarité. Paris, Presses Universitaires de France.

Sass C., Dupré C., Dauphinot V., Labbe E., Guéguen R., Gerbaud L. et Moulin J.J. (2005): The EPICES score: an individual index of material and social deprivation related to health status. Part II - Evaluation in a population of 197389 French subjects. Document disponible en ligne: http://www.cetaf.asso.fr/protocoles/precarite/protocoles_epices.htm Dernière consultation le 9 mai 2006.

- Singh-Manoux A. et Marmot M. (2005): Role of socialization in explaining social inequalities in health. Social Science et Medicine, Vol. 60, No 9, pp. 2129-2134

Sobel M. E. (1982). Asymptotic intervals for indirect effects in structural equations models. In S. Leinhart (Dir.), Sociological Methodology, San Francisco, Jossey-Bass, pp. 290-312.

- Sordes-Ader F. et TAP P. (2002): Précarité socio-économique et vulnérabilité. Pratiques Psychologiques, 4, pp. 65-78.

- Stein K. B., SARBIN T. R. et Kulik J. A. (1968): Future time perspective: its relation to the socialization process and the delinquent role. Fournal of Consulting and Clinical Psychology, Vol. 32, N ${ }^{\circ}$ 3, pp. 257264.

- WANBerg G. R. et Griffiths R. F. (1997): Time structure and unemployment: a longitudinal investigation. Fournal of Occupational and Organizational Psychology, Vol. 70, 이 1, pp. 75-96.

- Waters L. et Muller J. (2003): Money or time? Comparing the effects of time structure and financial deprivation on the psychological distress of unemployed adults. Australian Fournal of Psychology, Vol. 55, $\mathrm{N}^{\circ}$ 3, pp. 166-175.

-Zimbardo P. G. et Boyd J. N. (1999): Putting time in perspective: A valid, reliable individual-differences metric. Fournal of Personality and Social Psychology, Vol. 77, $\mathrm{N}^{\circ}$ 6, pp. 1271-1288. 


\section{Annexe}

Les 25 items de la version courte de la ZTPI construite à partir de la validation française.

\section{Dimension Passé-Positif $(n=7)$}

$\mathrm{N}^{\circ} 1:$ «Les images, les odeurs et les sons familiers de mon enfance me rappellent souvent des souvenirs merveilleux. »

$\mathrm{N}^{\circ} 3$ : « Le fait de penser à mon passé me donne du plaisir. »

$\mathrm{N}^{\circ} 7$ : «J'aime bien les histoires qui racontent comment les choses étaient au bon vieux temps. »

$\mathrm{N}^{\circ} 18$ : «Les souvenirs heureux des bons moments me viennent facilement à l'esprit. »

№ 20 : «Tout compte fait, ily a beaucoup plus de bonnes choses à se souvenir dans mon passé que de mauvaises. »

$\mathrm{N}^{\circ} 24$ : «J'ai la nostalgie de mon enfance »

No 25 : «f'aime bien les traditions et les coutumes familiales qui sont régulièrement répétées. »

Dimension Passé-Négatif $(n=5)$

$\mathrm{N}^{\circ} 4:$ : Il m’est difficile d'oublier des images désagréables de ma jeunesse »

$\mathrm{N}^{\circ} 6$ : «Je pense aux mauvaises choses qui me sont arrivées dans le passé»

$\mathrm{N}^{\circ} 9$ : «Je pense aux bonnes choses que j'ai ratées dans ma vie »

$\mathrm{N}^{\circ} 10$ : «Le passé comporte trop de souvenirs déplaisants auxquels je préfère ne pas penser »

$\mathrm{N}^{\circ} 13$ : «Les expériences douloureuses du passé me reviennent en permanence à l'esprit. »

\section{Dimension Présent-Fataliste $(n=4)$}

$\mathrm{N}^{\circ} 5$ : «Mon parcours dans la vie est contrôlé par des forces que je ne peux pas influencer.»

$\mathrm{N}^{\circ} 17$ : «Le destin détermine beaucoup de choses dans ma vie. »

$\mathrm{N}^{\circ} 19$ : «Puisque ce qui doit arriver arrivera, peut importe vraiment ce que je fais. »

ํo 22 : «Se préoccuper de l'avenir n'a aucun sens, puisque de toute façon je ne peux rien y faire. »

\section{Dimension Présent-Hédoniste $(n=3)$}

$\mathrm{N}^{\circ} 2$ : «Prendre des risques empêche ma vie de devenir ennuyeuse. »

$\mathrm{N}^{\circ} 16$ : «Fe prends des risques pour mettre de l'excitation dans ma vie. »

No 21 : «C'est important de mettre de l'excitation dans ma vie. »

\section{Dimension Futur $(n=6)$}

$\mathrm{N}^{\circ} 8$ : «Ffe fais aboutir mes projets à temps, en progressant étape par étape. »

$\mathrm{N}^{\circ} 11:$ «fe fais des listes de choses à faire. »

$\mathrm{N}^{\circ} 12$ : «Avant de se donner du bon temps le soir, mieux vaut penser à ce qu'il y a à faire pour le lendemain. »

$\mathrm{N}^{\circ} 14$ : «Avant de prendre une décision, je pèse le pour et le contre. »

$\mathrm{N}^{\circ} 15$ : «Quand je dois réaliser quelque chose, je me fixe des buts et j’envisage les moyens précis pour les atteindre. »

$\mathrm{N}^{\circ} 23$ : «Je crois que la journée d'une personne doit être planifiée à l'avance chaque matin. » 